\title{
Modulation of intertube band dispersion relation of carbon nanotube bundles by symmetry and intertube wave function coupling
}

\author{
Susumu Okada, ${ }^{\text {a) }}$ Yanlin Gao, ${ }^{\text {b) }}$ and Mina Maruyamac) \\ Department of Physics, Graduate School of Pure and Applied Sciences, \\ University of Tsukuba, 1-1-1 Tennodai, Tsukuba, Ibaraki 305-8571, \\ Japan
}

Based on density functional theory with a local density approximation and the simple tight-binding approximation, we investigated an electronic structure of carbon nanotube bundles in terms of mutual nanotube arrangement. The dispersion relation near the Fermi level along the intertube direction was found to be sensitive to the nanotube species and their mutual orientation within the bundles. Nanotube bundles with three-fold symmetry exhibited a substantial orientation dependence in the band dispersion relation along the intertube direction. The tight-binding calculation and wave function analysis revealed that this orientation dependence arises from the intertube wave function coupling and whether a node exists between nanotubes.

\footnotetext{
a)E-mail address: sokada@comas.frsc.tsukuba.ac.jp

b) E-mail address: ylgao@comas.frsc.tsukuba.ac.jp

c)E-mail address: mmaruyama@comas.frsc.tsukuba.ac.jp
} 


\section{INTRODUCTION}

Following the discovery of coaxial graphene tubules via transmission electron microscopy ${ }^{1}$, carbon nanotubes have held a dominant position among various nanoscale low-dimensional materials for three decades because of their unique geometric and electronic properties ${ }^{2-4}$. Early theoretical works reported that the electron states around the valence and conduction band edges of carbon nanotubes are sensitive to their diameter and chiral index $(n, m)$, leading to either a metallic or semiconducting electronic structure that corresponds with the discretization on the Dirac cone of graphene ${ }^{5-7}$. Furthermore, covalent tubular networks of three-fold-coordinated $\mathrm{C}$ atoms produce a remarkable mechanical toughness and stiffness ${ }^{8-11}$. Owing to these unique properties, carbon nanotubes are regarded as emerging materials in many modern technological areas such as flexible electrodes ${ }^{12-14}$, field effect transistors ${ }^{15-17}$, thermoelectric devices ${ }^{18-20}$, and reinforced materials ${ }^{8-11}$.

In addition to individual nanotubes, the tubular structure and chemically inert surfaces of carbon nanotubes also favor the formation of hierarchical structures with other nanotubes or foreign nanoscale materials, where constituent units are bound via van der Waals interaction, leading to further variation in their physical properties. Coaxial nanotubes are a representative example of such hierarchical structures and are known to possess unusual electronic structure that exceeded the simple sum of those of each constituent. Double-walled metallic nanotubes exhibit a small gap near the Fermi level caused by band repulsion, which is associated with the mutual orientation between inner and outer nanotubes ${ }^{21,22}$. Furthermore, double-walled semiconducting nanotubes exhibit a metallic electronic structure because the difference in wall curvatures introduces a difference in their work function ${ }^{23}$. Encapsulation of fullerenes and hydrocarbon molecules inside nanotubes has led to interesting hierarchical structures with mixed dimensionality that exhibit peculiar electronic structure modulations depending on the constituents and their arrangement within the nanotubes ${ }^{24-29}$.

Bundles of nanotubes are a simple extension of the structural hierarchy of nanotubes and are essential in various devices. Early theoretical calculations have predicted that interactions between nanotubes within bundles (i.e., intertube interactions) modulate the intrinsic electronic structure of the constituent nanotubes ${ }^{30-32}$. Recent advances in nanotube synthesis, purification, and assembly techniques have allowed experimental confirmation of the electronic structure modulation of highly-aligned homogeneous nanotubes via intertube in- 
teractions ${ }^{33-36}$. However, band dispersion relations along intertube direction of nanotube bundles are restricted in particular nanotubes and nanotube orientations.

In this work, we aim to investigate detailed electronic band structures of carbon nanotube bundles in terms of the nanotube species and its mutual orientation in the bundles, according to the above background, by performing first-principles total-energy calculations. Our

calculations based on the density functional theory (DFT) revealed that the electronic band structure along the intertube direction strongly depends on the symmetry of constituent nanotubes and their orientations. For bundles consisting of nanotubes with three-fold symmetry, the electronic structure across the nanotubes were highly dependent on the mutual orientation of the nanotubes. Tight-binding analyses revealed that this strong orientation dependence is owing to the intertube electron transfer, which arise from the mutual atomic arrangement between adjacent nanotubes. In contrast, the electronic structure of bundles comprising nanotubes without three-fold symmetry is insensitive to the nanotube orientation.

\section{CALCULATION METHODS AND STRUCTURAL MODELS}

The electronic and geometric structures of carbon nanotubes were investigated using $\mathrm{DFT}^{37,38}$ implemented in the program package STATE ${ }^{39,40}$. We used the local density approximation to describe the exchange-correlation potential energy among interacting electrons with the Perdew-Zunger functional form ${ }^{41,42}$. The interaction between electrons and ions is described using an ultrasoft pseudopotential ${ }^{43}$. The valence wave function and deficit charge density in the core region are expanded in terms of the plane wave basis sets with the cutoff energies of 25 and $225 \mathrm{Ry}$, respectively. Integration over the first Brillouin zone was carried out using equidistant $2 \times 2 \times 8 \boldsymbol{k}$-meshes in the directions along and lateral to the nanotubes, which were used for the self-consistent electronic structure calculations.

This work considered nanotube bundles consisting of $(9,9)$ and $(8,8)$ nanotubes. Within each bundle, the nanotubes were arranged in a triangular lattice with a nanotube spacing of $3.5 \AA$ (Fig. 1). The lattice parameter along tube axis was fixed as $2.459 \AA$, corresponding with the experimental bond length of graphene. For each bundle, the electronic structures were evaluated under several mutual orientations $\theta$. The atomic coordinates were optimized until the force acting on each atom was less than $1.33 \times 10^{-3} \mathrm{HR} / \mathrm{au}$ under the fixed lattice 

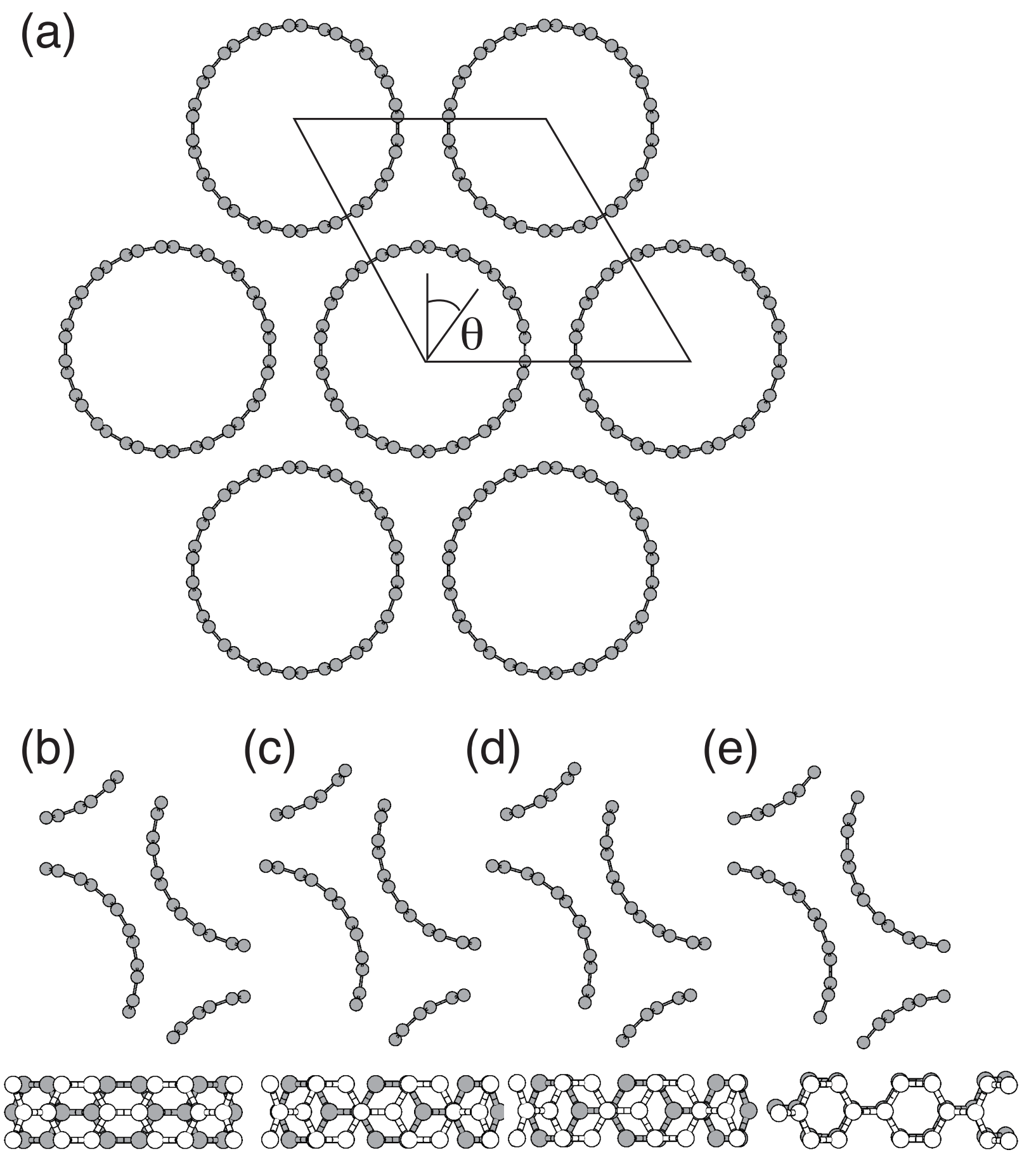

FIG. 1. (a) A geometric structure of $(9,9)$ nanotube bundles with mutual orientation angles $\theta$. Top and side views of detailed atomic arrangements between adjacent nanotubes in $(9,9)$ nanotube bundles with mutual angles of (b) $0^{\circ}$, (c) $3^{\circ}$, (d) $4^{\circ}$, and (e) $10^{\circ}$. A solid parallelogram indicates the unit cell. $\theta$ indicates the rotation angle of the nanotube. 
parameters in the directions along and normal to the carbon nanotubes.

\section{RESULTS}
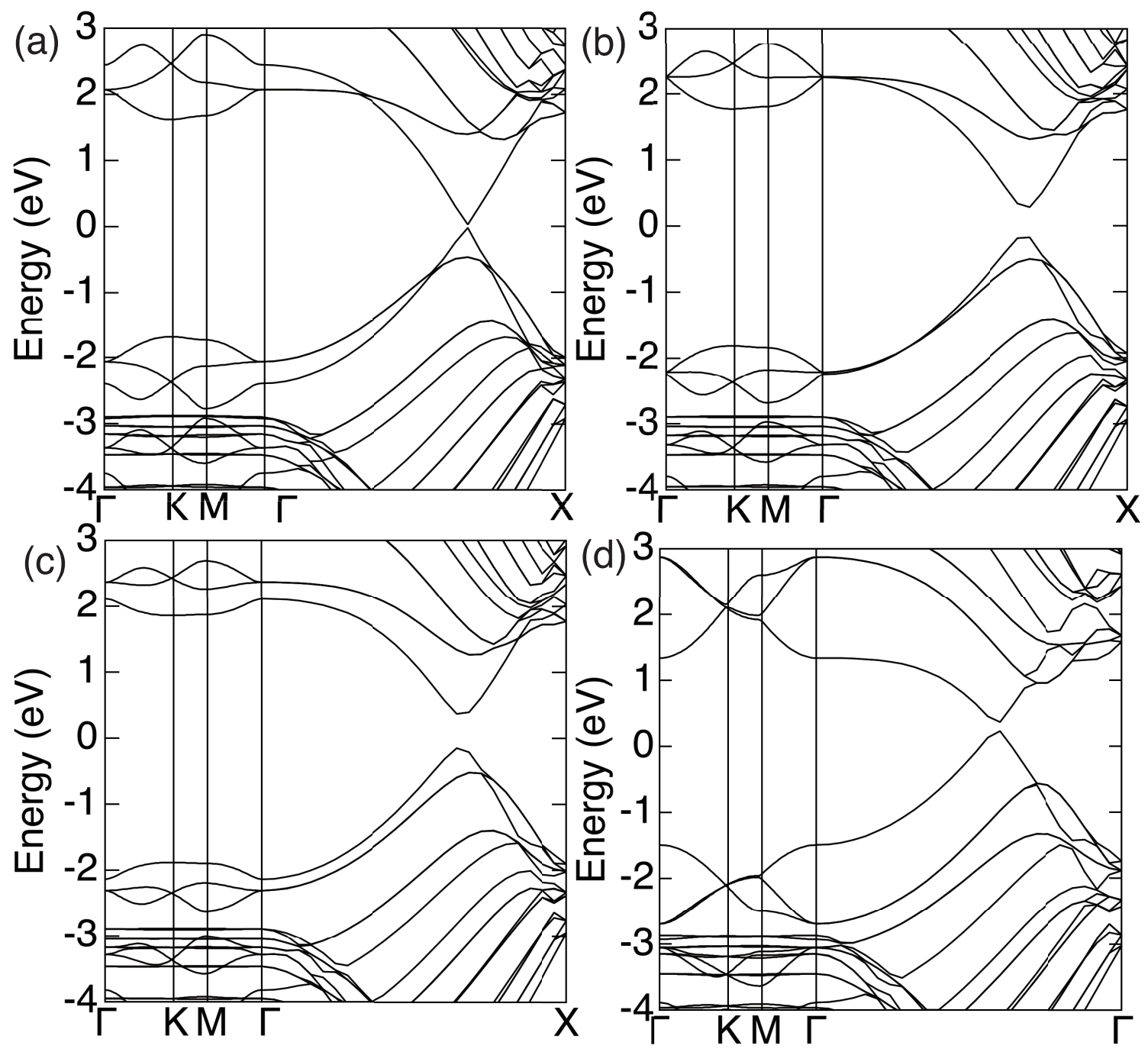

FIG. 2. Electronic energy band of $(9,9)$ nanotube bundles with mutual orientation angles $\theta$ of (a) $0^{\circ}$, (b) $3^{\circ}$, (c) $4^{\circ}$, and $10^{\circ}$. The energy is measured from the Fermi level.

Figure 2 shows the electronic structure of bundles comprising $(9,9)$ nanotubes with mutual orientation angles of $\theta=0,3,4$, and $10^{\circ}$. The dispersion relation along the intertube direction of the three highest branches of the valence band and the three lowest branches of the conduction band strongly depended on the nanotube angle $\theta$. For the nanotube bundle with 
$\theta=0^{\circ}$, the upper two branches of the valence band degenerated at the $\Gamma$ point while the lowest two crossed each other at the K point. Thus, these three branches exhibited a Kagome-like band structure comprising a less dispersive band and a dispersive band with a cone at the K point ${ }^{44,45}$. For the bundle with the angle of $3^{\circ}$, the three branches degenerated at the $\Gamma$ point, while the lower two of the three crossed each other at the K point. The three-fold degeneracy at the $\Gamma$ point was sensitive to the slight rotation of nanotubes: As the rotation angle slightly increased $\left(\theta=4^{\circ}\right)$, one of three branches was separated from the other two through the Brillouin zone with $k_{z}=0$. Furthermore, the band width was narrower than that with the other configurations. In contrast, for the angle of $10^{\circ}$, the dispersion relation of these three branches was highly modulated with a relation similar to that of the valence band of graphene with a $\sqrt{3} \times \sqrt{3}$ lateral unit cell. In addition, in this orientation the three branches possess the largest band width (i.e., $1.3 \mathrm{eV}$ ) among the four nanotube orientations. These results suggest that the nanotube arrangement induced a peculiar electronic band structure normal to the nanotubes, which is reminiscent of polymeric networks comprising hydrocarbon molecules ${ }^{44,45}$, even though the intertube spacing retains constant value for all angles. Note that the highest and lowest branches of valence and conduction bands of bundles with the rotation angles of 3,4 , and $10^{\circ}$ repulse each other around the $2 / 3$ of the $\Gamma-\mathrm{X}$ line as has been reported in the previous work ${ }^{30}$.
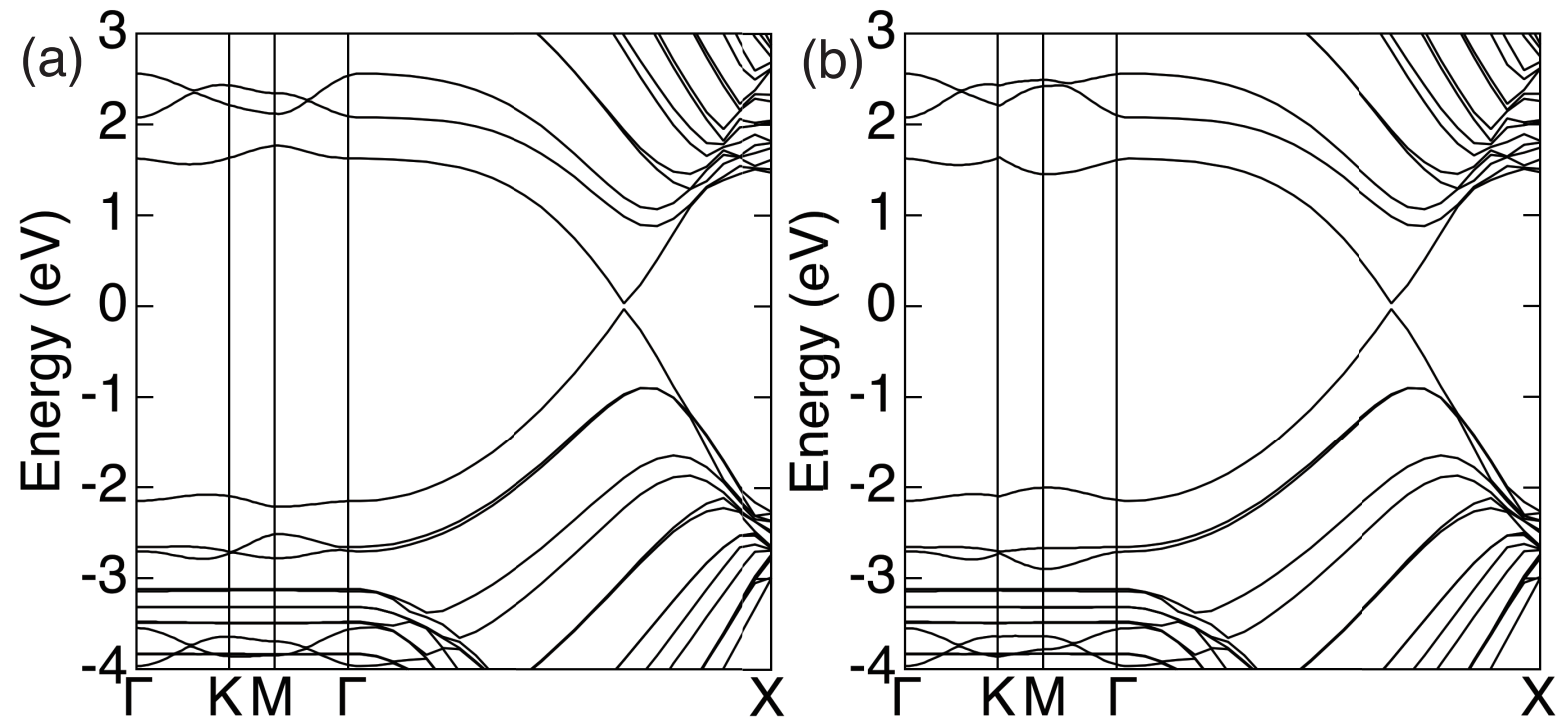

FIG. 3. Electronic energy band of $(8,8)$ nanotube bundles with mutual orientation angles $\theta$ of (a) $0^{\circ}$ and (b) $7^{\circ}$. The energy is measured from the Fermi level. 
Figure 3 shows the electronic structure of $(8,8)$ nanotube bundles with mutual orientation angles of $0^{\circ}$ and $7^{\circ}$. The three highest branches of the valence band and three lowest branches of the conduction band slightly depend on the mutual orientation in the bundles, unlike these same bands of the $(9,9)$ nanotube bundles. In particular, for both orientation angles, the highest branch of the valence band and the lowest branch of the conduction band are separated from the other two branches in their respective band with a remarkably small dispersion relation through the Brillouin zone with $k_{z}=0$. In addition to these isolated branches, the band widths of the remaining branches are also narrower in the $(8,8)$ nanotube bundle than the case of the $(9,9)$ nanotube bundle. These facts suggest that the commensurability between the bundle and the $(9,9)$ nanotube symmetries induced the peculiar orientation dependence of the electronic energy bands around the Fermi level along the intertube direction.

\section{DISCUSSION}

To investigate the physical origin of the orientation dependence of the electron states near the Fermi level, we performed the simple tight-binding calculation on the model structure shown in Fig. 4, thus simulating nanotube bundles with three-fold symmetry. Figure 5 shows the electronic energy band of the model structure under varying intertube and intratube electron transfers $t^{\prime}$ and $t$, respectively. For all cases, the upper band $(\varepsilon(\boldsymbol{k})>0$ : shaded area in Fig. 5) qualitatively reproduced the dispersion relation of the highest three branches of the valence band of the $(9,9)$ nanotube bundles. In particular, the DFT band of the bundle with the angle of $0^{\circ}$ [Fig. 2(a)] approximately agreed with the tight-binding band using the $t=-1$ and $t^{\prime}=0.9$ [Fig. 5(a)]; the DFT band of the bundle with the angle of $3^{\circ}$ [Fig. 2(b)] approximately agreed with the tight-binding band using the $t=-1$ and $t^{\prime}=0.5$ [Fig. 5(b)]; the DFT band of the bundle with the angle of $4^{\circ}$ [Fig. 2(c)] approximately agreed with the tight-binding band using the $t=-1$ and $t^{\prime}=0.2$ [Fig. 5(c)]; and the DFT band of the bundle with the angle of $10^{\circ}$ [Fig. 2(d)] approximately agreed with the tight-binding band using the $t=-1$ and $t^{\prime}=-0.9$ [Fig. 5(d)]. These results indicate that intertube transfer is crucial to determine the band dispersion relation of the nanotube bundle, indicating that the orientation dependence arises from the wave function overlap between adjacent nanotubes.

Using the band structure obtained with the tight-binding approximation, we investigated 


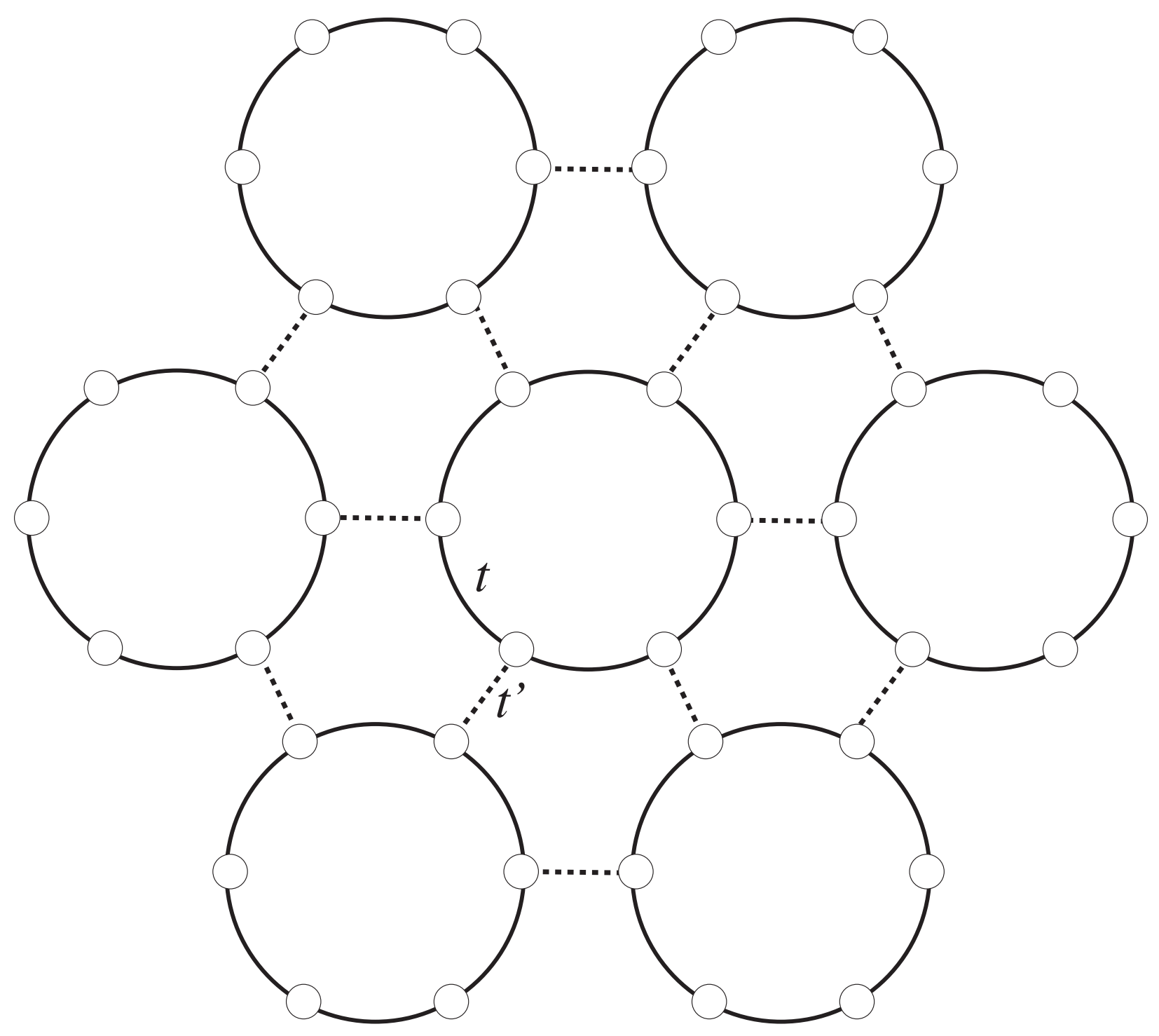

FIG. 4. Schematic structural model of the nanotube bundle where the nanotubes form a triangular lattice with intratube and intertube transfer integrals of $t$ (solid lines) and $t^{\prime}$ (dotted lines), respectively. Circles denote the atomic position of the nanotubes.

the wave function of one of the three highest branches of the valence band for each nanotube bundle (Fig. 6). For bundles with the angles of 0 and $3^{\circ}$, the wave function exhibited a bonding nature within the nanotube while exhibiting an antibonding nature between adjacent nanotubes [Figs. 6(a) and (b)]. This indicates that the wave functions of adjacent nanotubes are coupled in an opposite sign, as discussed in the previous paragraph. In contrast, for the bundles with the angle of $10^{\circ}$, the wave function exhibited a bonding nature both within and between nanotubes. These results show that the intertube wave 

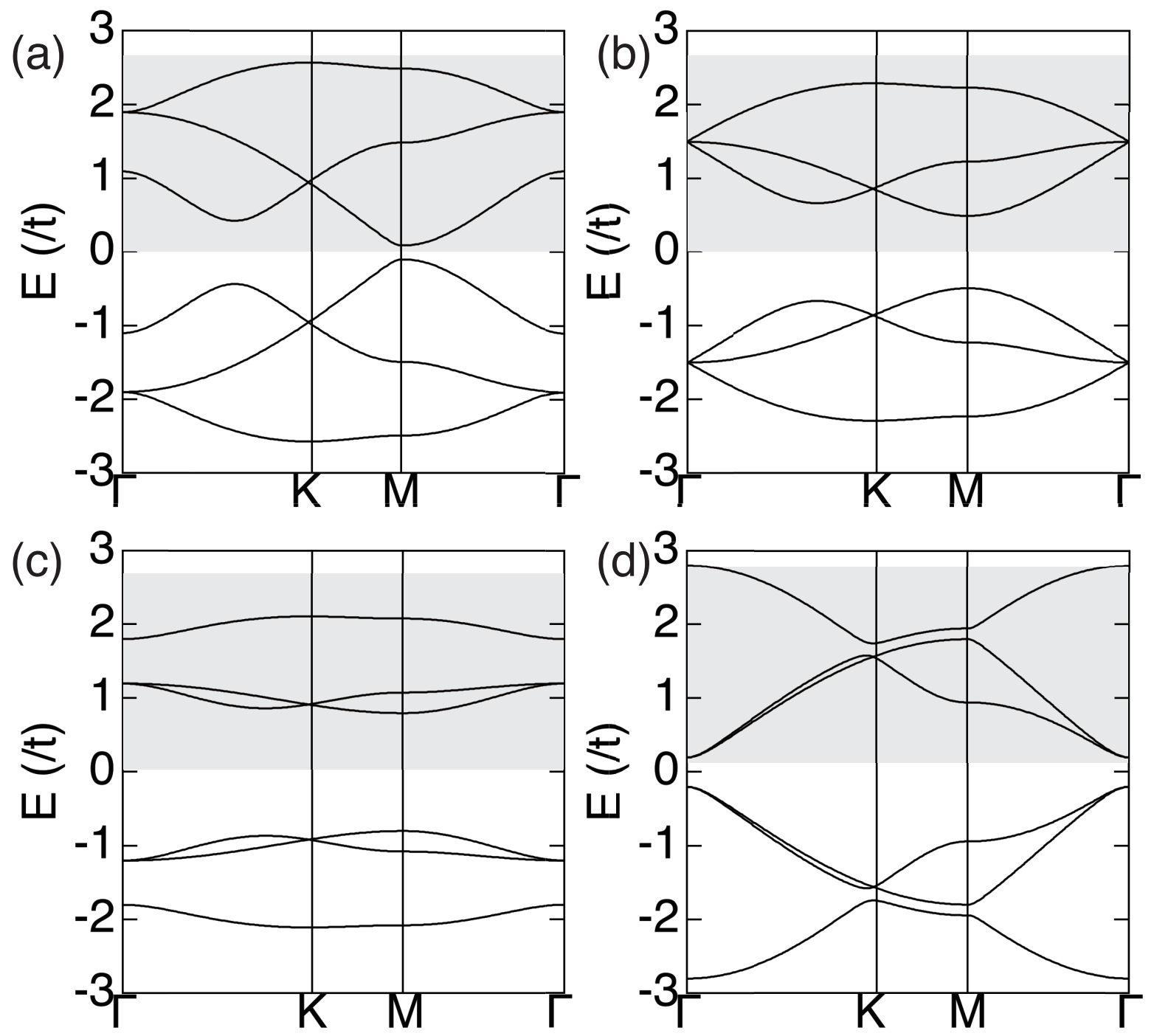

FIG. 5. Electronic energy band calculated by the simple tight-binding approximation for a structural model simulating the $(9,9)$ nanotube bundles in Fig. 4 with the tight binding parameters of (a) $t=-1$ and $t^{\prime}=0.9$, (b) $t=-1$ and $t^{\prime}=0.5$, (c) $t=-1$ and $t^{\prime}=0.2$, and (d) $t=-1$ and $t^{\prime}=-0.9$. Energy is in the units of the intratube transfer $t$. Shaded area indicate the upper energy bands possessing positive eigen values which qualitatively correspond with the highest three branches of the valence band normal to $(9,9)$ nanotube bundle in Fig. 2.

function coupling, which is determined by the atomic arrangements at the interfaces between adjacent nanotubes within bundles, will decisively affect the band dispersion relation along the intertube direction. This fact thus implies that the carrier distribution within nanotube bundles or thin films is greatly determined by the constituent nanotube species and their 


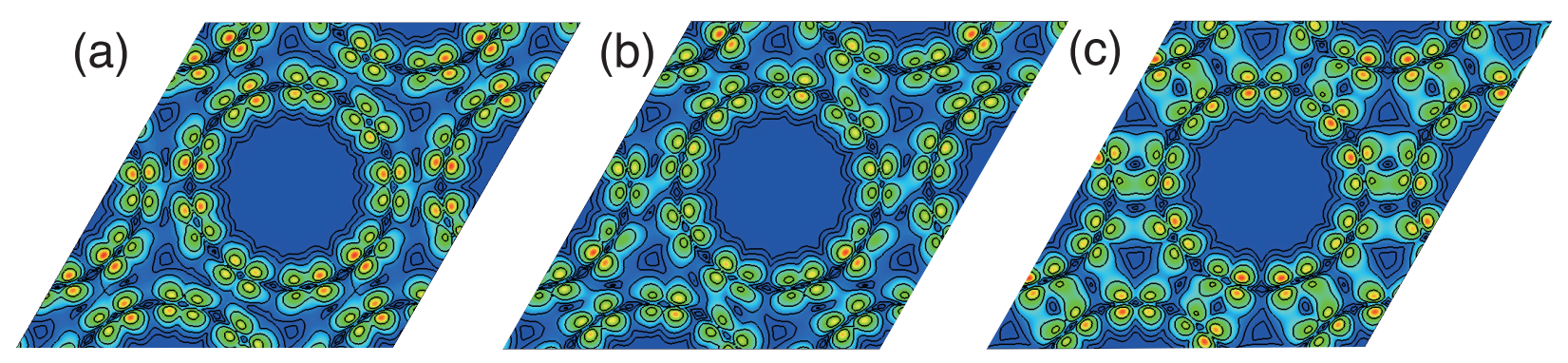

FIG. 6. Contour plot of the squared wave function of the highest branch of the conduction band at the $\Gamma$ point of nanotube bundles with a rotation angle of (a) $0^{\circ}$, (b) $3^{\circ}$, and $10^{\circ}$. Each contour curve is higher/lower density by double/half, respectively, than the adjacent contour curves. Indicated are regions of large (red) and small (blue) wave function amplitude.

arrangement, because the carrier penetration is determined by the intertube wave function hybridization ${ }^{46}$.

\section{CONCLUSION}

We investigated the electronic structure of carbon nanotube bundles using DFT and tight-binding approximation in terms of nanotube species and their mutual orientations. The electronic band structure along the intertube direction strongly depends on the symmetry of the constituent nanotubes and their orientations. In bundles consisting of nanotubes with three-fold symmetry, the energy band dispersion relation and band width of the three highest branches of the valence band and three lowest branches of the conduction band across the nanotubes are sensitive to the mutual orientation of the nanotubes. In contrast, the bundle comprising nanotubes lacking three-fold symmetry does not exhibit the orientation dependence in its band dispersion relation. The tight-binding analysis revealed that the orientation dependence arises from the intertube electron transfer between adjacent nanotubes. Wave function analysis of the energy band near the Fermi level indicates that the orientation sensitivity is caused by the existence or absence of a node between nanotubes. These results reveal the importance that the constituent nanotube species and their orientation within the bundle has on their effectiveness in their practical applications associated with the band edges. 


\section{ACKNOWLEDGMENTS}

The authors thank the Japan Science and Technology Agency, Core Research for Evolutionary Science and Technology (JST-CREST; Grant Nos. JPMJCR1532 and JPMJCR1715) and the Japan Society for the Promotion of Science, Grants-in-Aid for Scientific Research (JSPS KAKENHI; Grant Nos. JP20H00316, JP20H02080, JP20K05253, JP20H05664, and JP16H06331), the Joint Research Program on Zero-Emission Energy Research, Institute of Advanced Energy, Kyoto University, and the University of Tsukuba Basic Research Support Program (S). Part of the calculations were performed on an NEC SX-Ace at the Cybermedia Center at Osaka University.

\section{REFERENCES}

${ }^{1}$ S. Iijima, Nature 354, 56 (1991).

${ }^{2}$ M. S. Dresselhaus, G. Dresselhaus, and P. C. Eklund, Science of Fullerenes and Carbon Nanotubes (Academic Press, San Diego, CA,1996).

${ }^{3}$ M. S. Dresselhaus, G. Dresselhaus, and Ph. Avoris, Carbon Nanotubes Synthesis, Structure, Properties, and Applications (Springer, Berlin, 2001).

${ }^{4}$ S. Saito and A. Zettel Carbon nanotubes: Quantum cylinders of graphene (Elsevier, Amsterdam, 2008).

${ }^{5}$ N. Hamada, S.-I. Sawada, and A. Oshiyama, Phys. Rev. Lett. 68, 1579 (1992).

${ }^{6}$ R. Saito, M. Fujita, M. S. Dresselhaus, and G. Dresselhaus, Appl. Phys. Lett. 60, 2204 (1992).

${ }^{7}$ K. Tanaka, K. Okahara, M. Okada, and T. Yamabe, Chem. Phys. Lett. 191, 469 (1992).

${ }^{8}$ R. S. Ruoff and D. C. Lorents, Carbon 33, 925 (1995).

${ }^{9}$ M. M. J. Treacy, T. W. Ebbesen, and J. M. Gibson, Nature 381, 678 (1996).

${ }^{10}$ M.-F. Yu, O. Lourie, M. J. Dyer, K. Moloni, T. F. Kelly, and R. S. Ruoff, Science 287, $637(2000)$.

${ }^{11}$ M.-F. Yu, B. S. Files, S. Arepalli, and R. S. Ruoff, Phys. Rev. Lett. 84, 5552 (2000).

${ }^{12}$ I. Jeon, K. Cui, T. Chiba, A. Anisimov, A. G. Nasibulin, E. I. Kauppinen, S. Maruyama, and Y. Matsuo, J. Am. Chem. Soc. 1377982 (2015). 
${ }^{13}$ H.-Z. Geng, K. K. Kim, K. P. So, Y. S. Lee, Y. Chang and Y. H. Lee, J. Am. Chem. Soc. 129, 7758 ( 2007).

${ }^{14}$ B. Dan, G. C. Irvin and M. Pasquali, ACS Nano 3, 835 (2009).

${ }^{15}$ S. J. Tans, A. R. M. Verschueren, and C. Dekker, Nature 393, 49 (1998).

${ }^{16}$ R. Martel, T. Schmidt, H. R. Shea, T. Hartel, and Ph. Avouris, Appl. Phys. Lett. 73, 2447 (1998).

${ }^{17}$ Y. Nosho, Y. Ohno, S. Kishimoto, and T. Mizutani, Appl. Phys. Lett. 86, 073105 (2005).

${ }^{18}$ Y. Nakai, K. Honda, K. Yanagi, H. Kataura, T. Kato, T. Yamamoto, and Y. Maniwa, Appl. Phys. Express 7, 025103 (2014).

${ }^{19}$ K. Fukuhara, Y. Ichinose, H. Nishidome, Y. Yomogida, F. Katsutani, N. Komatsu, W. Gao, J Kono, and K. Yanagi, Appl. Phys. Lett. 113, 243105 (2018)

${ }^{20}$ Y. Ichinose, A. Yoshida, K. Horiuchi, K. Fukuhara, N. Komatsu, W. Gao, Y. Yomogida, M. Matsubara, T. Yamamoto, J. Kono, and K. Yanagi, Nano Lett. 19, 7370 (2019).

${ }^{21}$ Y.-K. Kwon and D. Tománek, Phys. Rev. B 58, R16001 (1998).

${ }^{22}$ Y. Miyamoto, S. Saito, and D. Tománek, 65, 041402R (2001).

${ }^{23}$ S. Okada and A. Oshiyama, Phys. Rev. Lett. 91, 216801 (2003).

${ }^{24}$ B. W. Smith, M. Monthioux, and D. E. Luzzi, Nature 396, 323 (1998).

${ }^{25}$ S. Okada, S. Saito, and A. Oshiyama, Phys. Rev. Lett. 86, 3835 (2001).

${ }^{26}$ M. Monthioux, Carbon 40, 1809 (2002).

${ }^{27}$ T. Okazaki, Y. Iizumi, S. Okubo, H. Kataura, Z. Liu, K. Suenaga, Y. Tahara, M. Yudasaka, S. Okada, and S. Iijima, Angew. Chem. Int. Ed. 50, 4853 (2011).

${ }^{28}$ S. Kigure, Y. Iizumi, T. Okazaki, and S. Okada, J. Phys. Soc. Jpn. 83, 124709 (2014).

${ }^{29}$ Y. Nagasawa, T. Koyama, and S. Okada, R. Soc. open sci. 5, 180359. (2018)

${ }^{30}$ P. Delaney, H. J. Choi, J. Ihm, S. G. Louie, and M. L. Cohen, Nature, 391, 466 (1998).

${ }^{31}$ P. Delaney, H. J. Choi, J. Ihm, S. G. Louie, and M. L. Cohen, Phys. Rev. B 60, 7899 (1999).

${ }^{32}$ S. Okada, A. Oshiyama, and S. Saito, Phys. Rev. B 62, 7634 (2000).

${ }^{33}$ M. Zheng, Top. Curr. Chem. 375, 13 (2017).

${ }^{34}$ Y. Yomogida, T. Tanaka, M. Tsuzuki, X. Wei, and H. Kataura, ACS Appl. Nano Mater. DOI: 10.1021/acsanm.0c02389

${ }^{35}$ Y. Ichinose, J. Eda, Y. Yomogida, Z. Liu, K. Yanagi, J. Phys. Chem. C 121, 13391 (2017). 
${ }^{36}$ Xi. He, W. Gao, L. Xie, B. Li, Q. Zhang, S. Lei, J. M. Robinson, E. H. Hároz, S. K. Doorn, W. Wang, R. Vajtai, P. M. Ajayan, W. W. Adams, R. H. Hauge, and J. Kono, Nat. Nanotechnol. 11, 633 (2016).

${ }^{37}$ P. Hohenberg and W. Kohn, Phys. Rev. 136, B864 (1964).

${ }^{38}$ W. Kohn and L. J. Sham, Phys. Rev. 140, A1133 (1965).

${ }^{39}$ Y. Morikawa, K. Iwata, and K. Terakura, Appl. Surf. Sci. 169-170, 11 (2000).

${ }^{40}$ Simulation Tool for Atom TEchnology, https://state-doc.readthedocs.io

${ }^{41}$ J. P. Perdew and A. Zunger, Phys. Rev. B 23, 5048 (1981).

${ }^{42}$ D. M. Ceperley and B. J. Alder, Phys. Rev. Lett. 45, 566 (1980).

${ }^{43}$ D. Vanderbilt, Phys. Rev. B 41, 7892 (1990).

${ }^{44}$ M. Maruyama, N. T. Cuong, and S. Okada, Carbon 109, 755 (2016).

${ }^{45}$ M. Maruyama and S. Okada, Carbon 125, 530 (2017).

${ }^{46}$ Y. Gao and S. Okada, J. Appl. Phys. 127, 134301 (2020) 